Conclusions: In the presentation we will show how and why the campaign could be such a success. We will focus on how to follow-up the positive results of generating leads and making invisible pain visible.

Disclosure of Interest: None declared

DOI: 10.1136/annrheumdis-2018-eular.2902

\section{OP0291-PARE RUNNING WITH RHEUMATISM: A 7-WEEK TRAINING PROGRAMME FOR NOVICE RUNNERS WITH INFLAMMATORY RHEUMATIC DISEASE}

J.F.M Holla ${ }^{1,2}$, J. Brontsema ${ }^{1}$, N. Schenk ${ }^{3}$, R. Jansen ${ }^{4}$, R. Vreenegoor ${ }^{5}$, M. Fluit ${ }^{1}$, W.F. Peter ${ }^{1}$, I.E. Bultink ${ }^{6}{ }^{1}$ Reade, Center for Rehabilitation and Rheumatology, Amsterdam; ${ }^{2}$ Faculty of Health, Sports and Social Work, Inholland University of Applied Sciences, Haarlem; ${ }^{3}$ Runner with inflammatory rheumatic disease, Weesp ${ }^{4}$ Club manager, Athletics Club Phanos, Amsterdam; ${ }^{5}$ Running trainer, Run2Day, Haarlem; ${ }^{6}$ Amsterdam Rheumatology and immunology Center, VU University Medical Center, Amsterdam, Netherlands

Background: In the Netherlands, running is the second most practised sport. Running is also becoming increasingly popular among people with inflammatory rheumatic diseases, according to the growing number of running-related questions to the Dutch Arthritis Foundation and rheumatologists. Frequently asked questions are: 'Am I allowed to run?' and 'How can I start in a safe way?'.

Objectives: Given the increasing demand from the target group, and the potential positive health consequences of high-intensity exercise, ${ }^{1}$ we developed a 7 week running programme for novice runners with inflammatory rheumatic disease, and explored its feasibility and safety.

Methods: First we performed a needs assessment among 228 participants with different inflammatory rheumatic diseases. In all, 200 (88\%) participants were interested in practicing the sport of running. Then, a rheumatologist, specialised physical therapist, patient representatives and experienced running trainers developed the running programme, based on the proven effective 'Start to Run' programme of the Dutch Athletics Federation. The programme aims to prepare participants in 7 weeks for a 20 min run without breaks, and consists of one supervised group training session and one or two non-supervised training sessions per week. In addition to gradual progression of joint load (by starting with running on soft even surfaces) and running volume, special attention is paid to development of muscle strength and coordination, optimal movement pattern and running at an own comfortable pace. During the programme, the running trainer is in close contact with a rheumatologist and/or specialised physical therapist who can be consulted for advice.

Results: Of the first 24 participants of 'Running with Rheumatism', 12 participants completed the programme successfully, 4 participants developed a running related injury, 1 participant dropped out due to rheumatic symptoms, and 7 participants dropped out due to other reasons (e.g. surgery, infectious mononucleosis, burn-out). Participants indicated that they felt safe and comfortable during the group training sessions, and were surprised to be able to achieve so much progress in a short time period. They felt that they had become stronger and got to know their body and physical capabilities better.

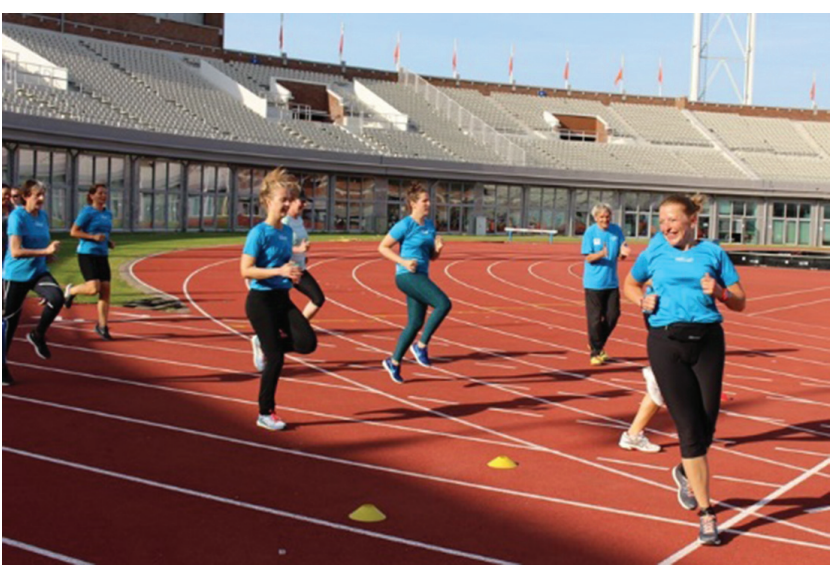

Conclusions: The first running groups were successful. The incidence of running related injuries did not significantly differ from incidence rates reported from comparable programmes for regular novice runners in the Netherlands. Therefore, we aim for nationwide implementation.

\section{REFERENCE:}

[1] Jong ZD, Munneke $M$, Zwinderman $A H$, et al. Is a long-term high-intensity exercise program effective and safe in patients with rheumatoid arthritis? Results of a randomized controlled trial. Arthritis \& Rheumatism 2003;48:2415-24.

Disclosure of Interest: None declared

DOI: 10.1136/annrheumdis-2018-eular.3782

\section{OP0292-PARE THE CREATION OF THE 'AQUA RE-ACTION'.A METHODOLOGICALLY BASED AQUATIC PHYSICAL INTERVENTION/REHABILITATION PROGRAM FOR PEOPLE WITH RMD'S}

\section{A. lacovou. Cyprus League Against Rheumatism, Limassol, Cyprus}

Background: As an Aquatic Instructor and an OA patient my self, I faced with other RMD patients the demand for an aquatic physical conditioning program that can meet our needs. After contacting with Patient Leagues and Aquatic Fitness Training Organisations, I found out that there was no such a specific program and it was essential to create one.

Objectives: My first objective was to get trained and create an aquatic program that could improve the quality of life through improving the ROM, muscular strenth flexibility, balance and aerobic capacity. My next objective was to accomodate people with variables like having or not fear of water, with minimum or increased joint mobility, with different affected joints and connective tissues. Hence to that had to approach and convince Rheumatologists, Orthopaedics, Physiotherapists and patients for the benefits of this specific Aquatic program and have it as an option to their rehabilitation. Moreover, I had to use water as my basic instrumen and use it' s principles (Laws of physics) to estimate the programs intensity. Finally I decided to offer that knowledge through an Instructor course.

Methods: In order to get trained, I followed specific training in Aquatic fitness and Rehabilitation by International Aquatic Organisations like the Aquatic Exercise Association, International Aquatic Therapy Faculty and BECO Academy and spending hours in the pool testing exercises and designing the program.

Methodogically, I implemented the Principles of Aquatic Environment like buoyancy, hydrostatic pressure, inertia, and biomechanics like, hydrodynamics, movement speed, levers, to estimate the exercise in low to medium intensity and I emphasised on the the quality and the execution of the movement. The program was focused on no pain moves in the biggest possible ROM, using all joints and muscle groups, working on muscle flexibility strength and aerobic capacity. I created a shallow and a deep water program in order to classify participants based on certain criteria

I contacted meetings with Physicians, and the Cyprus League Against Rheumatism. Published article about Aquatic workout benefits to RMD's at the Cyprus League Against Rheumatism (Cyplar) Journal, I gave lectures and special offer to Cyplar members.

The creation of the Instructors course, needed to start from registering a name, logo and manual.It was also essential to built a business structure to obtain financial and human resources

Results: Based on the participants testimonies there was an improvement on quality of life, their joints range of motion, muscle strength, flexibility and aerobic capacity. Hence to that they mention less pain, move quality night sleep and generally better social life with out depression. All the above was mentioned also from their Physicians and therapists that lead to increasing their trust towards the program benefits. Hence to that our collaboration with CYPLAR continued by organising a joined annual Swimming charity event.

AQUA Re-Action is the registered name of the program, and Logo and Manual are in an ongoing process of final registrations.

Conclusions: Having an active lifestyle through an aquatic physical conditioning program, which is under the guidance of a specialised Aquatic Instructor, for at least 3 times per week, can lead to a significant improvement on patients Quality of life within few weeks.

Disclosure of Interest: None declared

DOI: 10.1136/annrheumdis-2018-eular.1674 